\title{
Simulating a high frequency piezo pump with disc reed valves
}

\author{
Nathan Sell, Andrew Plummer, Nigel Johnston, and Jonathan du Bois \\ Department of Mechanical Engineering, University of Bath, Bath, United Kingdom \\ E-mail: N.P.Sell@bath.ac.uk
}

\begin{abstract}
Piezo pumps provide an attractive alternative for driving small actuators (e.g. less than $100 \mathrm{~W}$ ) compared to traditional valve controlled cylinders powered by a central hydraulic supply. This provides the ability to distribute power electrically rather than hydraulically, which can bring both weight and efficiency savings. Currently the use of piezo pumps is severely limited by the maximum power and flows that can be provided. This paper documents the simulation of a new pump which makes use of disc type reed valves to rectify the flow generate by a single piezostack-driven piston. The proposed valves have the potential to overcome frequency limitations of more conventional poppet or ball type check valves. This enables the pump to operate at higher frequencies and thereby produce larger flows. Simulation results suggest that a pump capable of producing a no load flow in excess of $1 \mathrm{~L} / \mathrm{min}$ would be possible using an off-the-shelf piezo stack.
\end{abstract}

Keywords: Piezopump, micro-hydraulics, piezoelectrics, reed valve

\section{Introduction}

The application of an electrical field to a piezoelectric ceramic results in a strain being induced in the material. The magnitude of this strain depends on the specific piezoceramic strain coefficient and also the strength of the applied field. In practical applications this is around 0.1 to $0.15 \%$ [1]. Piezoelectric stacks consist of multiple layers of ceramic and electrodes and are commercially available with total lengths up to around 200mm giving strokes of around $300 \mu \mathrm{m}$. The face area of the piezoceramic governs the force which it is capable of applying, and stacks with diameters of over $50 \mathrm{~mm}$ are commercially available giving maximum forces of up to $70 \mathrm{kN}$. This combination of high force and low stroke presents challenges in many applications which wish to take advantage of the small size, high reliability and lack of magnetic influence of piezoceramic actuators.

To overcome the stroke limitations various means of motion accumulation have been proposed. There are a number of commercially available mechanical accumulation methods including the PI walking drive (up to $125 \mathrm{~mm}$ stroke and $0.75 \mathrm{~W}$ output) [2], Noliac amplified actuators (upto $0.95 \mathrm{~mm}$ stroke and $0.8 \mathrm{~W}$ output) [3] and other methods currently being researched [4]. All these actuators generally have a maximum output power of less that 5W however meaning they are not competitive with even the smallest traditional hydraulic drives..

The piezoelectric pump concept (commonly contracted to "piezopump"), uses hydraulic motion accumulation instead of mechanical and has been shown to be capable of delivering larger powers. There are a number of different designs for piezopumps but all have a similar operating principle. A piezoelectric stack is used to drive a piston (or diaphragm) and a pair of valves are used to control the flow into and out of the piston chamber. Cycling the piston an high frequencies the small displacement can still result in appreciable amounts of flow. Figure 1 shows the operating cycle of a piezopump.

The operating cycle is made up of 4 stages. The first of these is the compression stage with the piezostack extending and compressing the fluid in the pumping chamber. When the pressure in the chamber is equal to that at the pump outlet the exhaustion stage begins as the outlet valve opens and any further extension of the piezostack is used to push fluid from the pumping chamber providing flow. Once the stack has reached the end of its stroke and begins retracting the expansion stage begins. As the stack retracts the pressure in the chamber drops towards the pressure at the inlet valve. When these pressures are equal the intake stage begins with the inlet valve opening and allowing fluid flow into the pumping chamber. As shown in Figure 1 there is often an accumulator on the pump inlet to both 
1. Compression

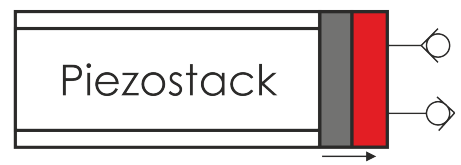

3.Expansion

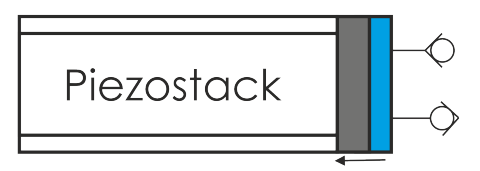

2. Exhaust

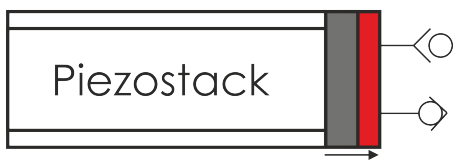

4. Intake

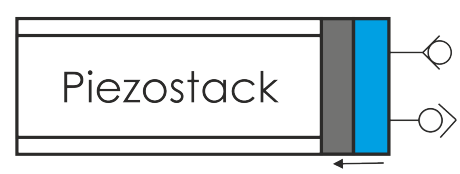

Figure 1: Operating principle of a piezopump

help with volume changes in the closed system (e.g. due to heating) and raise the net pressure of the circuit above atmospheric pressure in order to avoid cavitation at the inlet.

Small, low flow piezopumps (in the order of $\mathrm{mL} / \mathrm{s}$ ) are commercially available for applications such as medical dispensing [5] but currently only one commercially available unit with appreciable flow is know to exist [6].

An excellent review of piezopump technology was published by Chaudhuri and Wereley [7] in 2012. They suggest that most designs have 4 key limitations:

1. Actuator mechanical stiffness - if stiffer actuators were available then it should be possible to reduce the diameter of the actuators and still achieve the same blocking forces and thus reduce the size of piezopumps.

2. Fluid inertia and compressiblity - as the driving frequency increases the inertia of the fluid becomes more important as it must be accelerated and decelerated each cycle. Given the small strokes associated with piezoactuators the compressiblity of the working fluid also significantly limits pump performance as a considerable portion of the actuator stroke can be used compressing the fluid.

3. Bandwidth of driving circuits - performance may be limited by the ability of power electronics to provide full voltage at high frequencies.

4. Flow rectification using valves - Chaudhuri and Wereley argue that it is necessary to move towards active valves in order to increase operating frequency and therefore performance.

Since 2012 there have been a number of significant research papers including [8-15] These papers suggest that instead of a movement to active valves as predicted by [7] researchers are starting to investigate reed valves and the possibility of using multiple discrete pumping units in order to achieve higher flows. The power electronics often proved to be a limiting factor suggesting that co-development of the pump and driver is likely to be required in order to significantly increase power.

This paper focuses on the simulation of a piezopump which features disc type check valves for both its inlet and outlet, and an off-the-shelf piezostack The expected flow and pressure characteristics from such a device and frequency behaviour are predicted.

\section{Piezopump modelling}

\subsection{Piezostack modelling}

A Kinetic Ceramics D075080 stack is used in this simulation and its main parameters are given in Section 2.1.

The size was limited in order that commonly available amplifiers would be capable of driving the stack for future experimental work. The existence of hysteresis in piezoactuators is well documented and is simulated using the Bouc-Wen model [16] due to its tractability and ability to model a wide range of different hysteretic behaviours, Equation (1) shows this model where $\alpha, \beta$ and $\gamma$ are tuneable parameters and $x$ and $z$ are the input and output respectively.

$$
\dot{z}=a \dot{x}-\beta|\dot{x}||z|^{n-1} z-\gamma \dot{x}|z|^{n}
$$




\begin{tabular}{ll}
\hline Cross-sectional area & $0.0003 \mathrm{~m}^{2}$ \\
Length & $87 \mathrm{~mm}$ \\
Maximum voltage & $1000 \mathrm{~V}$ \\
Free displacement $\left(x_{\max }\right)$ & $80 \mu \mathrm{m}$ \\
Blocking force $\left(F_{\text {block }}\right)$ & $10 \mathrm{kN}$ \\
Capacitance & $1.77 \mu \mathrm{F}$ \\
Natural Frequency & $18 \mathrm{kHz}$ \\
\hline
\end{tabular}

A 10\% effective voltage loss was assumed and the parameters set accordingly. The voltage available to drive the stack is converted to a force via a simple gain of $10 \mathrm{~N} / \mathrm{V}$, and the force acting on the stack from the piston (due to pressure in the pump chamber) is subtracted from this force giving the total force available to extend the stack. The stack is then treated as a mass spring damper system with stack stiffness calculated as:

$$
K_{\text {piezo }}=\frac{F_{\text {block }}}{x_{\max }}
$$

The stack is assumed to be critically damped (when fluid and seal damping are taken into account) and have an effective mass equal to $40 \%$ of the stacks actual mass [17]. The piston mass is added to this effective mass

The general form of the piezostack model is shown in Figure 2

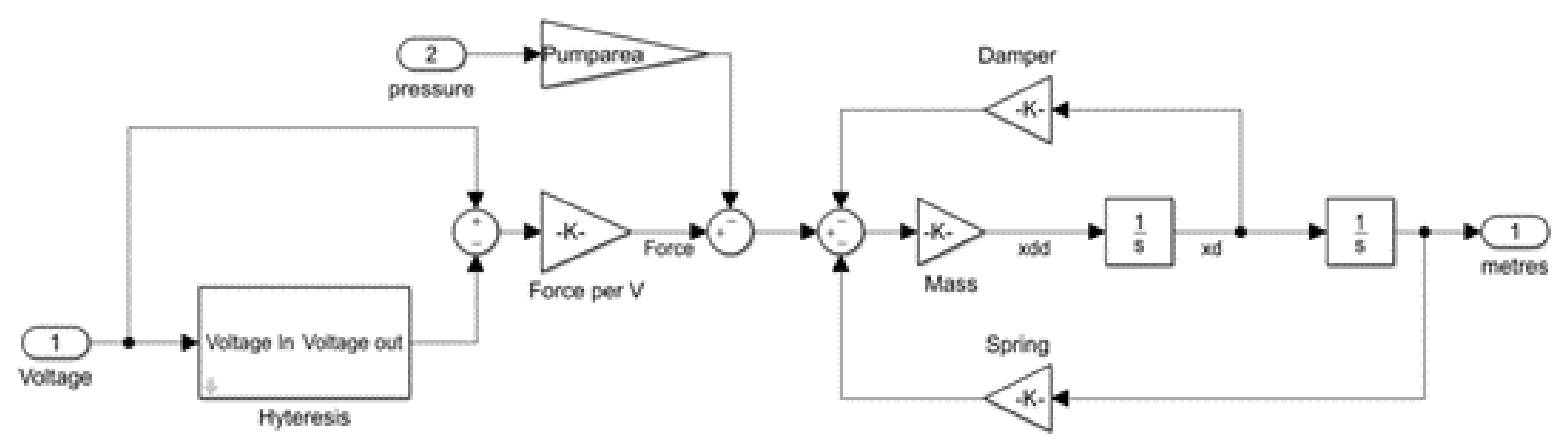

Figure 2: Piezoelectric actuator model

\subsection{Pump chamber modelling}

The displacement of this stack is assumed to be equal to the displacement of the piston. The sizing of the piston in piezopump is a trade off between flow and pressure performance. In the first instance the piston diameter will be assumed equal to the stack diameter but an exploration of its effect will be carried out. Multiplying the displacement of the stack by the area of the piston gives a volume change in the pumping chamber. This is combined with the volume change due to flow through the inlet $\left(Q_{i n}\right)$ and outlet $\left(Q_{\text {out }}\right)$ valves to give a chamber volume change $\left(\Delta Q_{c h}\right)$ which can be multiplied by the pump stiffness $\left(K_{\text {pump }}\right)$ to give a chamber pressure:

$$
\begin{gathered}
\Delta V_{c h}=x \cdot A_{p}-\int Q_{o u t}+\int Q_{i n} \\
P_{c h}=K_{\text {pump }} \cdot \Delta V_{c h}
\end{gathered}
$$

The pump stiffness is a combination of the fluid stiffness $K_{\text {fluid }}$, piezostack stiffness $K_{\text {piezo }}$ and pump body radial $K_{r a}$ and axial $K_{a x}$ stiffness: 


$$
\begin{aligned}
K_{\text {fluid }} & =\frac{B}{V_{c h}} \\
K_{\text {piezo }} & =\frac{k_{\text {piezo }}}{A_{p}^{2}} \\
K_{r a} & =\frac{4 E \cdot t}{\pi D_{c h}^{3} L} \\
K_{a x} & =\frac{E \pi D_{c h} t}{L A_{c h}^{2}} \\
K_{\text {total }} & =\frac{1}{\frac{1}{K_{\text {fluid }}}+\frac{1}{K_{\text {piezo }}}+\frac{1}{K_{r a}}+\frac{1}{K_{a x}}}
\end{aligned}
$$

where $B$ is the bulk modulus of the working fluid and assumed to be $1.2 \mathrm{GPa}, V_{c h}$ is the initial volume of the pumping chamber which is another design trade-off that must be considered. Small chambers result in stiffer pumps (and therefore better performance) but they can also cause manufacturing or assembly difficulties and in the case of extremely small chambers severe flow restrictions from to the inlet and outlet valves. A chamber height of $5 \mathrm{~mm}$ is chosen to balance these competing requirements. $E$ is the Youngs modulus of the chamber wall material (assumed to be 207GPa for mild steel), $t$ is the wall thickness (assumed to be 15mm)

\subsection{Check Valve Modelling}

The pressure in the pumping chamber dictates the opening on the passive disc reed valves whose flow rates are referenced in Equation (3). Currently it is assumed that the inlet and outlet check valves are identical and they are modelled to include the effects of valve and fluid inertia, valve bounce and context dependent opening times. The models are based on previous work conducted at Bath [18] for poppet type valves. Although disc reed valves will be used, experimentally derived coefficients are currently required in detailed reed valve models [19] making the models unsuitable for early investigations. It is proposed that a single-degree of freedom model with lumped mass and stiffness parameters is adequate, so that the disc reed valve can be treated in the same way as a poppet valve. 3 shows the diagram of the passive poppet type valve model.

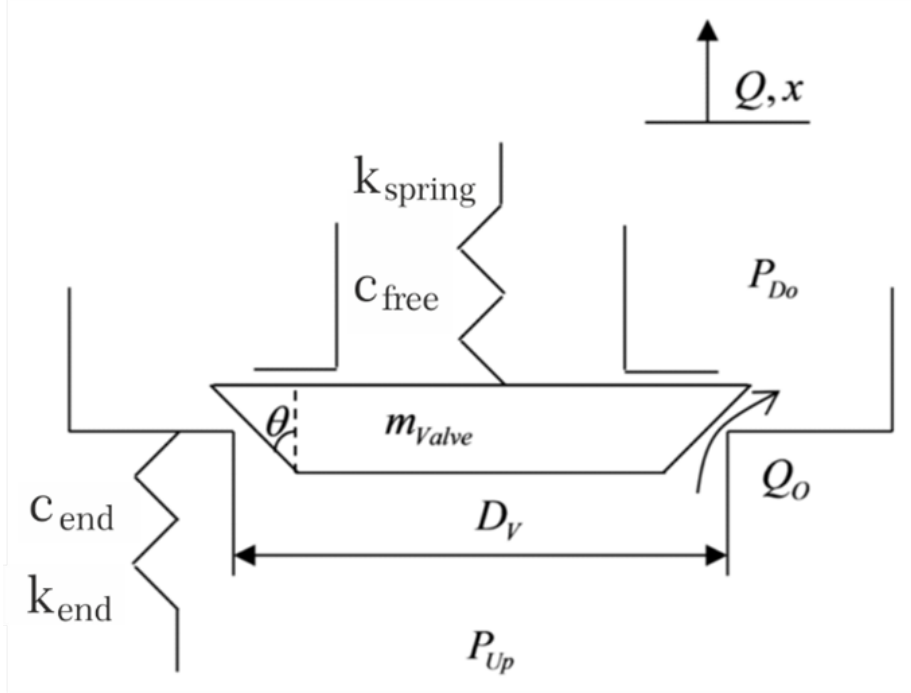

Figure 3: Check valve model [18]

The valve has a mass $\left(m_{\text {valve }}\right)$ and is closed by a spring with linear stiffness $\left(k_{s p r i n g}\right)$ and there is an associated damping $\left(c_{\text {free }}\right)$ :

$$
c_{\text {free }}=2 \zeta \sqrt{k_{\text {spring }} m_{\text {valve }}}
$$

When the valve reaches the end stop it is subject to a high stiffness $\left(k_{\text {stop }}\right)$ and damping $\left(c_{\text {stop }}\right)$ also changes as would be expected from Equation (10). The net force accelerating the the valve mass $\left(F_{\text {valve }}\right)$ is a combination of 
the spring and damper forces and the pressure difference across the valve face area.The net force can take one of three forms dependent upon the poppets position:

$$
\begin{aligned}
& \text { Case } 1: \text { valve fullyopen }\left(x \geq x_{\text {stop }}\right) \\
& F_{\text {valve }}=A_{\text {valve }} C_{f} \Delta P-c_{\text {stop }} \dot{x}-k_{\text {stop }}\left(x-x_{\text {stop }}\right)-k_{\text {spring }} x-F_{\text {pre }}
\end{aligned}
$$

Case 2 : valve partiallyopen $\left(0<x<x_{\text {stop }}\right)$

$$
F_{\text {valve }}=A_{\text {valve }} C_{f} \Delta P-c_{\text {free }} \dot{x}-k_{\text {spring }} x-F_{\text {pre }}
$$

$$
\begin{aligned}
& \text { Case } 3: \text { valve fullyclosed }(x \leq 0) \\
& F_{\text {valve }}=A_{\text {valve }} C_{f} \Delta P-c_{\text {stop }} \dot{x}-k_{\text {stop }} x-k_{\text {spring }} x-F_{\text {pre }}
\end{aligned}
$$

where $A_{\text {valve }}$ is the valve face area, $C_{f}$ is the valve force coefficient and is a function of valve design and is fixed at 0.4 and $F_{\text {pre }}$ is the preload force on the valve. Using this force the position of the check valve can be found from integrating:

$$
\ddot{x}=\frac{F_{\text {valve }}}{m_{\text {valve }}+m_{\text {fluid }}}
$$

where $m_{\text {fluid }}$ is the "added mass" due to the fluid displaced as the valve moves. The flow through the valve is:

$$
Q=Q_{o}+A_{\text {valve }} \cdot \dot{x}
$$

where $Q_{o}$ is the flow rate through the valve orifice, $A_{\text {valve }}$ is the valve face area and $\dot{x}$ is the velocity of the valve. A value for $Q_{o}$ can be found by integration from the pressure change due to fluid inertia:

$$
\Delta P_{v}=\frac{\rho}{2} \cdot\left(\frac{Q_{o}}{C_{d} \pi A_{v}}\right)^{2}+\frac{\rho l}{a} \frac{d Q_{o}}{d t}
$$

where $\Delta P_{v}$ is the pressure difference across the valve and so for the inlet valve is the pressure in the piston chamber minus the pressure at pump inlet, $\rho$ is the fluid density (taken to be $850 \mathrm{~kg} / \mathrm{m}^{3}$ ), $C_{d}$ is the valve discharge coefficient which is a function of valve design and assumed to be $0.72, l$ is the characteristic length of the fluid, and assumed to be the thickness of the valve and $a$ is the valve opening area. An opening area lower limit of $1 \mathrm{~mm}^{2}$ is set to model leakage and aid mathematical stability.

\subsection{Reed Valve modifications}

The actual form of the valve is shown in Figure 4.

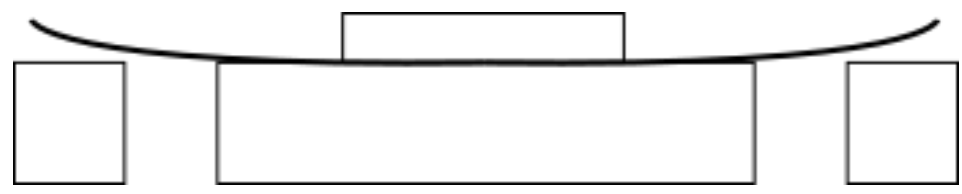

Figure 4: Disc type reed valve cross section

The valve opening area above with be calculated as:

$$
a=\pi \cdot d_{\text {orifice }} \cdot x_{\text {valve }}
$$

where $x_{\text {valve }}$ is defined as the displacement of the disc at the outer edge of the annulus. The discs mass is a function of its diameter:

$$
m_{\text {valve }}=\frac{1}{3} A_{\text {valve }}\left(\rho_{\text {valve }} \cdot t_{\text {valve }}+\rho_{\text {fluid }} \cdot x_{\text {valve }}\right)
$$


It is assumed that the deformation of the disc results in a triangular cross section and so the effective mass of the valve is a third of the valves mass plus the mass of fluid displaced by the valves motion. The stiffness of the disc can also be related to the dimensions of the valve by using Timoshenko's bending equation for a ring [20]. In order to apply this equation simplifications are made. Firstly it is assumed that all the opening force is located at the centre of the annulus. This is reasonable when the valve is closed. Secondly it is assumed that the centre of the ring is clamped. The tip displacement can then be calculated from:

$$
x_{\text {valve }}=k_{1} \frac{P d_{\text {orifice }}^{2}}{2 \cdot E t_{\text {valve }}^{3}}
$$

where $k_{1}$ is a coefficient dependent on the boundary conditions and ratio of valve diameter to hole diameter, $E$ is the Young's modulus (assumed to be 207GPa). Using this displacement and the load force a stiffness can be found.

\section{Sensitivity of pump performance to valve parameters}

In order to investigate the effects of valve geometry it was initially assumed that an outer diameter equal to the piston diameter $(19.5 \mathrm{~mm})$ and inner diameter of $6 \mathrm{~mm}$ to allow for fastening of the valve to a seat and a thickness of $100 \mu \mathrm{m}$. It was assumed that the disc valves would be manufactured by chemical etching and as such they diameter of the disc could be easily adjusted but the thickness would be limited to standard sheet thicknesses.

Figure 5 shows the power delivered by the pump with different valve thicknesses. In order to measure this a small volume is connected to the pump's outlet and and the pressure and flow through a $5 \times 10^{-5} \mathrm{~m}^{2}$ orifice connecting this volume back to the pump inlet is then calculated. The piezostack is excited by a $1 \mathrm{kV}$ sine wave at $1 \mathrm{kHz}$.

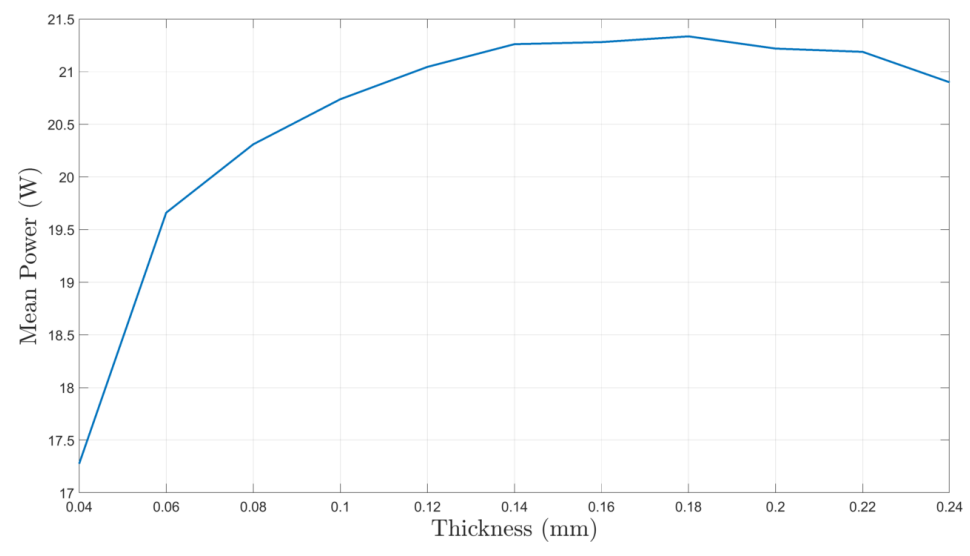

Figure 5: Effect of valve thickness on flow

A peak of $21 \mathrm{~W}$ was found at $0.16 \mathrm{~mm}$ with performance being reasonably consistent between $0.14 \mathrm{~mm}$ and $0.22 \mathrm{~mm}$. As mentioned previously the valve thickness changes both stiffness and mass of the valve meaning the optimal flow conditions will be influenced by the operating frequency also. Operating the pump at $1 \mathrm{kHz}$ as this is sufficiently below the undamped natural frequency of the valve whilst also being in advance of frequencies attainable using traditional poppet style check valves. Looking at the effect of the valve outer diameter, Figure 6.

suggests that for a specific operating frequency there should exist an optimum set of valve dimensions and so a constrained optimisation was conducted on the valve thickness, inner and out diameters. This was carried out using the Matlab fmincon functions implementation of the interior-point algorithm [21]. The outer diameter was limited to $19 \mathrm{~mm}$ in order to ensure the chamber volume was not increased and the ratio of inner diameter to outer diameter constrained to remain inside the range found in [20]. The minimum valve thickness was $0.1 \mathrm{~mm}$ to ensure it could be etched from a standard sheet of metal which from Figure 5 is expected to be in the optimum range. Figure 7 shows the iteration history of this optimisation procedure where the aim is it maximise the mean pump power over its complete operating range from free flow to dead-headed.

The optimisation gave valve parameters of:

$$
\begin{aligned}
d_{\text {valve }} & =12.3 \mathrm{~mm} \\
h_{\text {valve }} & =4.1 \mathrm{~mm} \\
t_{\text {valve }} & =100 \mu \mathrm{m}
\end{aligned}
$$




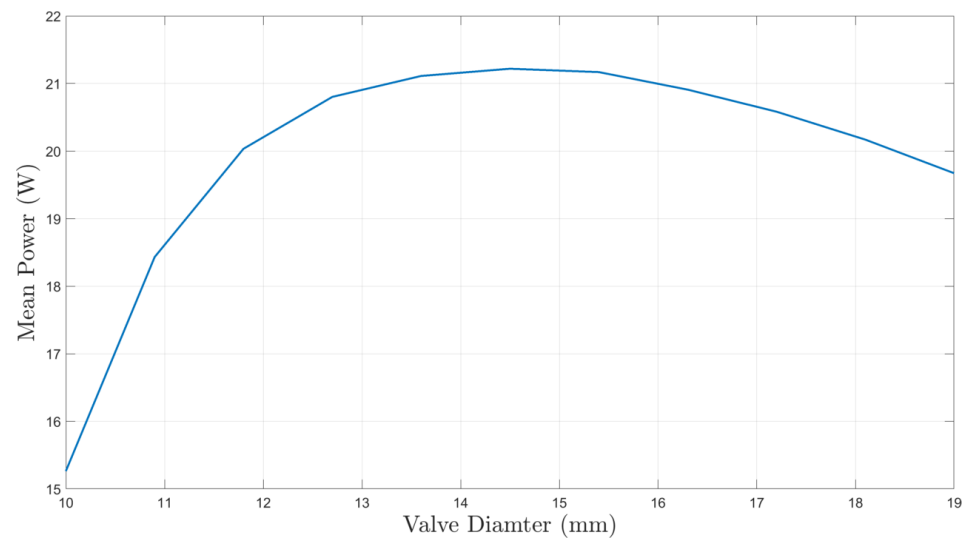

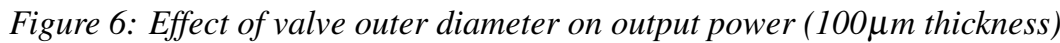

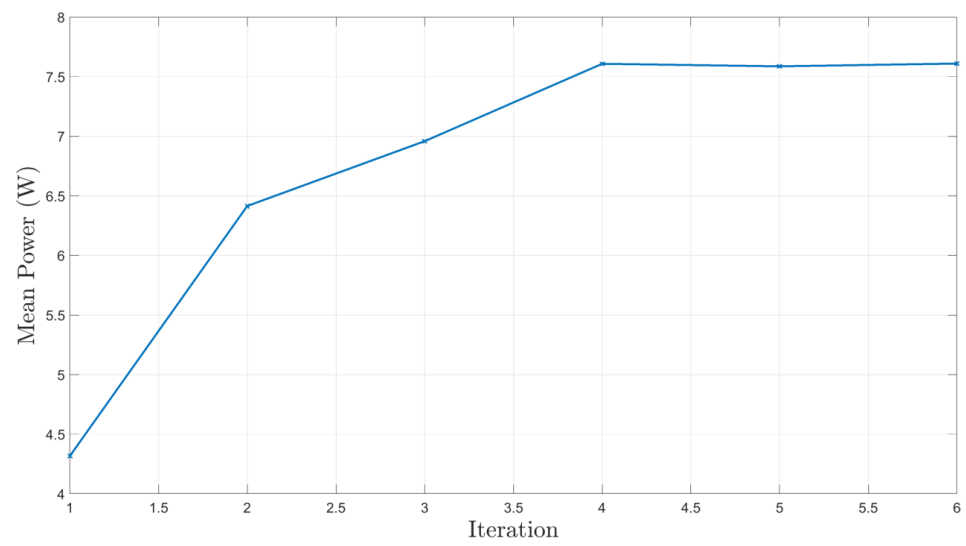

Figure 7: Optimisation of disc valve parameters 


\section{Performance of pump with optimised parameters}

The simulated performance of the pump with these valve parameters at a range of driving frequencies and outlet pressures is shown in Figure 8.

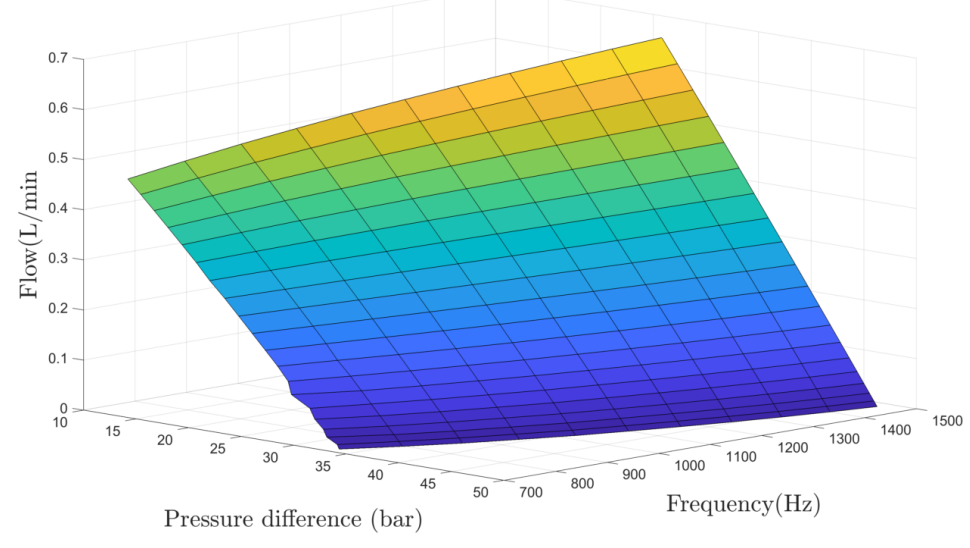

Figure 8: Simulated pressure flow behaviour of pump

As expected, the flow reduces with increasing pressure, due to reduction in stack displacement with increased force, and the need to overcome higher compressibility flow and the increase in performance as frequency increases drops off as the frequency itself increases. However, there is still some improvement in performance all the way up to $1500 \mathrm{~Hz}$ suggesting that the valve bandwidth exceeds this.

\section{Conclusions}

The use of disc reed check valves offers a promising avenue to extend the operating frequency of piezo pumps, allowing them to be considered for higher power applications than previously possible. As a simple model for this style of valve does not exist, one is proposed based on previous work on poppet check valves and Timoshenko ring bending. The results of pump simulations using this model are promising, however, the model hasn't been validated and simplifies the bending of the valve into a simple tip deflection with no allowance for the deformed shape and so the exact numbers should be regarded with caution. However, it is clear that valve parameters such as disc diameter and thickness have a significant effect on pump performance. The flexibility and ease of prototyping given by chemical etching enables optimal disc valves to be manufactured for a particular size of pump. Preliminary experimental results confirm that this type of valve enables piezo pump operation at $1 \mathrm{kHz}$ or above; these results will be presented in a future publication. 


\section{References}

[1] Choi SB and Han YM. Piezoelectric actuators: control applications of smart materials. CRC Press, 2016.

[2] LTD PI. Piezowalk actuators. https://www.physikinstrumente.co.uk/en/products/ linear-actuators/. Accessed: 2020-04-08.

[3] Noliac. Amplified actuators. http://www.noliac.com/products/actuators/ amplified-actuators/. Accessed: 2020-04-08.

[4] Tian X, Quan Q, Wang L et al. An inchworm type piezoelectric actuator working in resonant state. IEEE Access 2018; 6: 18975-18983.

[5] Microfluidics D. Piezoelectric pump. https://www.dolomite-microfluidics.com/product/ piezoelectric-pump/. Accessed: 2020-04-08.

[6] Ceramics K. Solid-state pumps. https://www.kineticceramics.com/products/ solid-state-pumps/. Accessed: 2020-07-15.

[7] Chaudhuri A and Wereley N. Compact hybrid electrohydraulic actuators using smart materials: A review. Journal of intelligent material systems and structures 2012; 23(6): 597-634.

[8] Larson JP and Dapino MJ. Reliable, high-frequency miniature valves for smart material electrohydraulic actuators. Journal of intelligent material systems and structures 2012; 23(7): 805-813.

[9] Xuan Z, Jin T, Ha NS et al. Performance of piezo-stacks for a piezoelectric hybrid actuator by experiments. Journal of intelligent material systems and structures 2014; 25(18): 2212-2220.

[10] Li Y, Le VT, Goo NS et al. High actuation force of piezoelectric hybrid actuator with multiple piezoelectric pump design. Journal of Intelligent Material Systems and Structures 2017; 28(18): 2557-2571.

[11] Zhang Z, Chen S, Wang S et al. Performance evaluation and comparison of a serial-parallel hybrid multichamber piezoelectric pump. Journal of Intelligent Material Systems and Structures 2018; 29(9): 19952007.

[12] Hwang JH, Bae JS, Hwang YH et al. Pressurization characteristics of a piezoelectric-hydraulic pump for uav brake systems. International Journal of Aeronautical and Space Sciences 2018; 19(3): 776-784.

[13] Nguyen AP, Hwang JH, Hwang YH et al. Effect of check valve characteristics on flow rate of the small piezoelectric-hydraulic pump. Journal of Aerospace System Engineering 2018; 12(5): 54-68.

[14] Woo J, Sohn DK and Ko HS. Experimental study on pressure pulsation in piezo driven reed valve pump. Journal of Mechanical Science and Technology 2019; 33(2): 661-667.

[15] Woo J, Sohn DK and Ko HS. Performance and flow analysis of small piezo pump. Sensors and Actuators A: Physical 2020; 301: 111766.

[16] Wen YK. Method for random vibration of hysteretic systems. Journal of the engineering mechanics division 1976; 102(2): 249-263.

[17] Fox J and Mahanty J. The effective mass of an oscillating spring. American Journal of Physics 1970; 38(1): 98-100.

[18] Johnston DN. Numerical modelling of reciprocating pumps with self-acting valves. Proceedings of the institution of mechanical engineers, Part I: Journal of systems and control engineering 1991; 205(2): 87-96.

[19] Knutson AL and de Ven James D V. Modeling and experimental validation of disc and reed style check valves for hydraulic applications. In Bath/ASME 2016 SYmposium on Fluid Power and Motion Control. ASME.

[20] Timoshenko SP and Woinowsky-Krieger S. Theory of plates and shells. McGraw-hill, 1959.

[21] Byrd RH, Hribar ME and Nocedal J. An interior point algorithm for large-scale nonlinear programming. SIAM Journal on Optimization 1999; 9(4): 877-900. 